\title{
Ab initio study of noble gas atoms in bcc transition metals
}

Chao Jiang, Yongfeng Zhang, Yipeng Gao, Jian Gan

June 2018

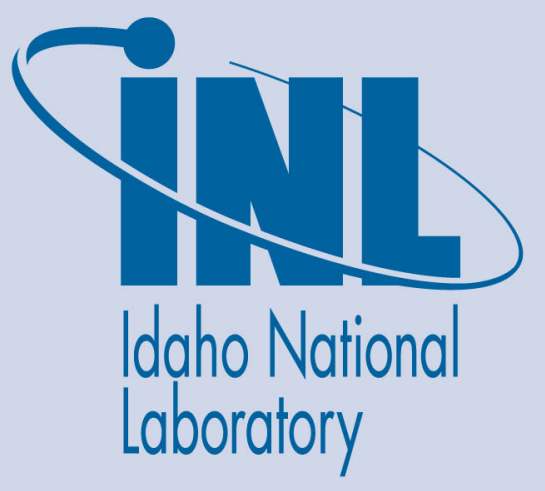

The INL is a U.S. Department of Energy National Laboratory operated by Battelle Energy Alliance 


\title{
Ab initio study of noble gas atoms in bcc transition metals
}

\author{
Chao Jiang, Yongfeng Zhang, Yipeng Gao, Jian Gan
}

June 2018

Idaho National Laboratory Idaho Falls, Idaho 83415

http://www.inl.gov

Prepared for the

U.S. Department of Energy

Under DOE Idaho Operations Office

Contract DE-AC07-05ID14517 


\title{
$A b$ initio theory of noble gas atoms in bec transition metals
}

\author{
Chao Jiang, ${ }^{\text {*a }}$ Yongfeng Zhang, ${ }^{a}$ Yipeng Gao ${ }^{\mathrm{a}}$ and Jian Gan ${ }^{\mathrm{b}}$ \\ ${ }^{\mathrm{a}}$ Fuels Modeling and Simulation, Idaho National Laboratory, Idaho Falls, ID 83415, USA \\ b Advanced Characterization Department, Idaho National Laboratory, Idaho Falls, ID 83415, USA
}

\begin{abstract}
Systematic ab initio calculations based on density functional theory have been performed to gain fundamental understanding of the interactions between noble gas atoms ( $\mathrm{He}, \mathrm{Ne}, \mathrm{Ar}, \mathrm{Kr})$ and bcc transition metals in group 5B $(\mathrm{V}, \mathrm{Nb}, \mathrm{Ta}), 6 \mathrm{~B}(\mathrm{Cr}, \mathrm{Mo}, \mathrm{W})$ and $8 \mathrm{~B}(\mathrm{Fe})$. Our charge density analysis indicates that the strong polarization of nearest-neighbor metal atoms by noble gas interstitials is the electronic origin of their high formation energies. Such polarization becomes more significant with increasing gas atom size and interstitial charge density in the host bcc metal, which explains the similar trend followed by the unrelaxed formation energies of noble gas interstitials. Upon allowing for local relaxations, nearby metal atoms move farther away from gas interstitials in order to decrease polarization, albeit at the expense of increasing elastic strain energy. Such atomic relaxations are found to play an important role in governing both the energetics and site preference of noble gas atoms in bcc metals. Our most notable finding is that the fully relaxed formation energies of noble gas interstitials are strongly correlated with the elastic shear modulus of the bcc metal, and the physical origin of this unexpected correlation has been elucidated by our theoretical analysis based on the effective-medium theory. The kinetic behavior of noble gas atoms and their interaction with pre-existing vacancies in bcc transition metals have also been discussed in this work.
\end{abstract}

*Corresponding author: chao.jiang@inl.gov 


\section{Introduction}

Understanding the interactions between noble gas atoms and metals is of both scientific and technological interests, and has long been an active area of research. Due to their closed-shell electron configurations, the equilibrium solubility of noble gases in metals is extremely low under ambient conditions. Only at exceedingly high pressures (e.g. in Earth's core), noble gases such as helium start to exhibit some chemical reactivity and form stable compounds with metals. ${ }^{1}$ Despite their low thermodynamic solubility, noble gas atoms can be forced into a metal lattice under non-equilibrium conditions, e.g., during high-energy ion bombardment., Furthermore, in a nuclear reactor, helium can be produced internally in a metal lattice by neutron-induced $(n, \alpha)$ transmutation reactions. The trapped noble gas atoms strongly tend to precipitate out as gas bubbles ${ }^{2-4}$ and lead to void swelling, surface blistering, and significant degradation of properties such as thermal conductivity and mechanical strength, which is a great concern for the safety and reliability of nuclear reactors. Under certain experimental conditions, nanoscale gas bubbles can self-organize into ordered three-dimensional superlattices with a body-centered cubic (bcc) or face-centered cubic (fcc) structure. ${ }^{2-4}$ The presence of a gas bubble superlattice is believed to be beneficial to irradiation tolerance by helping the metal lattice maintain a stable swelling behavior during irradiation. ${ }^{5}$

A fundamental understanding of the nucleation and growth of noble gas bubbles in metals and their self-organization into ordered three-dimensional superlattices is of great importance for the design of structural materials for current fission and future fusion nuclear reactors. To this end, detailed knowledge of the stable lattice configurations, stabilities and 
mobilities of noble gas atoms and their clusters is critical. Such atomistic-scale information is however often difficult to measure experimentally. During the past decades, atomistic simulations based on either empirical potentials ${ }^{6-8}$ or density functional theory (DFT) $)^{9-16}$ have been widely used to provide this missing information. These studies provide key properties related to gas bubble formation and represent an important initial step towards multi-scale modeling of noble gas effects in materials.

The purpose of this work is to use systematic DFT calculations to gain fundamental understanding of the thermodynamic behavior of noble gas atoms in bcc transition metals, which are technologically important materials for building next-generation nuclear reactors. For example, $\mathrm{W}$ is a promising plasma-facing material for fusion reactors due to its high melting point, low sputtering yield, and high thermal conductivity. ${ }^{8,11,14} \mathrm{Fe}-\mathrm{Cr}$-based ferritic-martensitic steels are also appealing fuel cladding materials for fast reactors due to their excellent resistance to void swelling. ${ }^{17}$ Without allowing for local relaxations, the formation energies of noble gas interstitials are extremely large due to the strong polarization of nearest-neighbor metal atoms. The unrelaxed formation energy depends on both the unperturbed interstitial charge density of the host metal and the size of the noble gas atom. Upon atomic relaxations, the polarization of metal atoms is reduced due to increased gas-metal separation, although at the cost of increased elastic strain energy. By decomposing the total formation energy into electronic and elastic contributions, we have developed a theoretical model based on the effective-medium theory ${ }^{18-20}$ to fully consider the local relaxation effects. Our most notable finding is the unexpectedly strong correlation between the fully relaxed formation energy of a noble gas interstitial and the shear modulus of the host bcc metal. This indicates that the energetics of noble gas atoms in bcc 
transition metals are more strongly dominated by the local relaxation process rather than the initial electronic interactions between a gas atom and a perfect metal lattice. The kinetic behavior of noble gas atoms and their interaction with irradiation-induced vacancies have also been discussed in this work.

\section{Computational methods}

DFT calculations are performed using the generalized gradient approximation of Perdew, Burke, and Ernzerhof (PBE-GGA) $)^{21}$ as implemented in the Vienna ab initio Simulation Package. ${ }^{22} \mathrm{~A}$ large 250 -atom $5 \times 5 \times 5$ cubic bcc supercell is employed to minimize the unphysical electronic and elastic interactions between a defect and its periodic images. The electron-ion interactions are described by the projector-augmented wave (PAW) method. ${ }^{23}$ The semi-core $3 p$ electrons of V and $\mathrm{Cr}$, the $4 p$ electrons of $\mathrm{Nb}$ and $\mathrm{Mo}$, and the $5 p$ electrons of $\mathrm{Ta}$ and $\mathrm{W}$ are explicitly treated as valence electrons. The cutoff energy for plane-wave basis sets is set at $350 \mathrm{eV}$. For Brillouin zone sampling, a $3 \times 3 \times 3$ Monkhorst-Pack $k$-point mesh is used for the 250 -atom supercells. Our convergence tests indicate that such choice of cutoff energy and $k$-point mesh is sufficient to give fully converged results. By computing the quantum mechanical Hellmann-Feynman forces, all cell-internal atomic positions are fully optimized using the conjugate-gradient scheme until the forces acting on atoms are less than $0.01 \mathrm{eV} \AA^{-1}$. The unit cell volume and shape are hold fixed during structural relaxations. Spin-polarized calculations are performed to account for the ferromagnetic nature of Fe. For Cr, the nonmagnetic state is assumed in our calculations since it is only slightly higher in energy than the antiferromagnetic state. ${ }^{24}$ We define the formation energy of a gas atom $\mathrm{X}$ at interstitial and substitutional positions in metal $\mathrm{M}$ as: 


$$
E_{\text {int }}^{f}=E\left(M_{N} X_{1}\right)-N E_{M}-E_{X}
$$

and

$$
E_{s u b}^{f}=E\left(M_{N-1} X_{1}\right)-(N-1) E_{M}-E_{X}
$$

where $N$ is the number of metal atom sites in the perfect bec supercell. $E_{\mathrm{M}}$ is the energy per atom of the bcc metal. $E_{\mathrm{X}}$ is the energy of an isolated $\mathrm{X}$ atom calculated by placing it in a $20 \times 20 \times 20$ $\AA^{3}$ cell. Finally, the binding energy between a vacancy and an interstitial gas atom can be calculated as:

$$
E_{X_{\mathrm{int}}-V a}^{\text {bind }}=E_{\mathrm{int}}^{f}+E_{V a}^{f}-E_{\text {sub }}^{f}
$$

where $E_{V a}^{f}$ is the vacancy formation energy. Here a positive and negative binding energy indicates attraction and repulsion between entities, respectively.

\section{Results and discussion}

\subsection{Tetrahedral vs. octahedral site preference}

Table 1 reports the formation energies of noble gas atoms occupying tetrahedral (Fig. 1(a)) and octahedral (Fig. 1(b)) interstitial positions in bec transition metals from both our unrelaxed and relaxed calculations. In the former, all metal atoms are fixed at their ideal lattice positions. The difference between the unrelaxed and relaxed results thus gives the energy gained by relaxation of metal atoms upon the incorporation of a gas atom. Interestingly, according to our unrelaxed calculations, all noble gas atoms strongly prefer tetrahedral over octahedral sites in all bcc 
transition metals despite the larger free volume of the latter. From Fig. 2, it can be seen that the unrelaxed formation energy generally increases with both increasing size of the noble gas atom $(\mathrm{He}<\mathrm{Ne}<\mathrm{Ar}<\mathrm{Kr})$ and the unperturbed charge density at the interstitial region of the host metal. For a given noble gas species, its unrelaxed formation energies in group $6 \mathrm{~B}(\mathrm{Cr}, \mathrm{Mo}, \mathrm{W})$ metals are also decidedly larger than those in group $5 \mathrm{~B}(\mathrm{~V}, \mathrm{Nb}, \mathrm{Ta})$ metals.

To gain some insight into the electronic origin of these trends, we plot in Figs. 3 and 4 the change in charge density in bcc transition metals upon insertion of a noble gas atom at tetrahedral and octahedral site, respectively. No relaxation of metal atoms is allowed. Here the charge density difference is defined as $\Delta \rho=\rho\left(M_{N} X_{1}\right)-\rho\left(M_{N}\right)-\rho_{X}$. Here $\rho\left(M_{N} X_{1}\right)$ and $\rho\left(M_{N}\right)$ represent the charge density of a perfect metal lattice with and without a gas atom, respectively. Blue contours indicate regions of charge depletion while red contours represent charge accumulation. Evidently, a noble gas atom at tetrahedral (octahedral) site strongly polarizes its four (two) first-nearest-neighbor metal atoms, and such polarization becomes more significant with increasing gas atom size and interstitial charge density in the host metal. In comparison, the polarization of the four second-nearest-neighbor metal atoms of a gas atom at octahedral position is much less pronounced, which can be understood by the fact that their distance from the gas atom $\left(0.707 a_{0}\right)$ is $41 \%$ greater than that of the first-nearest-neighbor metal atoms $\left(0.5 a_{0}\right)$. Here $a_{0}$ is the bcc lattice constant. Importantly, the increase in unrelaxed formation energy with increasing gas atom size and host metal charge density can be well explained by the corresponding increase in the energetically unfavorable polarization of nearby metal atoms. Compared with group 5B metals, more significant polarization is observed in group 
$6 \mathrm{~B}$ metals due to their higher interstitial charge densities. Presumably, this is the electronic origin of the larger unrelaxed formation energies of noble gas atoms in the latter group. Since the polarization diminishes rapidly with increasing gas-metal separation, the shorter gas-metal distance for an octahedral interstitial $\left(0.5 a_{0}\right)$ than a tetrahedral interstitial $\left(0.559 a_{0}\right)$ also explains why unrelaxed calculations consistently predict the tetrahedral site as the energetically preferred one for noble gas atoms.

Upon allowing for local relaxations, nearby metal atoms move farther away from the gas atom in order to decrease polarization. As shown in Table 1, such relaxations can significantly reduce the formation energy of a noble gas atom by as much as $75 \%$ at a tetrahedral site and $79 \%$ at an octahedral site. Since the relaxation energy for an octahedral interstitial is always much greater than that of a tetrahedral interstitial, the energy difference between the two configurations is drastically reduced. Remarkably, for $\mathrm{Ne}, \mathrm{Ar}$ and $\mathrm{Kr}$ in group 5B metals, the relaxation energies are even large enough to reverse the tetrahedral vs. octahedral site preference. Consequently, while He prefers to occupy tetrahedral sites in all bcc transition metals, $\mathrm{Ne}, \mathrm{Ar}$ and $\mathrm{Kr}$ favor octahedral sites in group 5B metals but tetrahedral sites in bcc metals in groups $6 \mathrm{~B}$ and $8 \mathrm{~B}$. Our fully relaxed formation energies also agree well with previous DFT studies in the literature. ${ }^{9-16}$

To quantitatively understand the effects of local relaxations, it is worthwhile to separate the total gas atom formation energy into electronic and elastic contributions as:

$$
E_{\text {int }}^{f}(x)=E_{\text {elastic }}(x)+E_{\text {electronic }}(x)
$$


where $x$ is the displacement of first-nearest-neighbor metal atoms surrounding an interstitial site away from their ideal lattice positions (see insets of Fig. 5(a)), which measures the degree of local relaxations. For octahedral site, the four second-nearest-neighbor metal atoms are expected to be displaced less than $x$ since they are farther away from the gas atom. An empirical ratio of $3 / 8$ is extracted from our fully relaxed DFT geometries. $E_{\text {elastic }}$ is the increase in strain energy when a metal lattice is locally deformed in order to increase the free volume of an interstitial site and to make room for gas atom incorporation. In computing elastic energies, the four (six) metal atoms immediately surrounding a tetrahedral (octahedral) interstice are fixed at positions according to a given displacement $x$, while all other metal atoms in the supercell are allowed to fully relax. $E_{\text {electronic }}$ is the energy needed to embed a gas atom at an interstitial site and is due to the repulsive interaction between a noble gas atom and host metal electron density. While $E_{\text {elastic }}$ will increase with increasing $x, E_{\text {electronic }}$ will decrease with $x$ since atomic relaxations will increase gas-metal separation and consequently reduce the polarization of metal atoms. As illustrated in the inset of Fig. 5(b), the final relaxed formation energy is determined by a close competition between elastic and electronic contributions.

To answer the question why He atom prefers tetrahedral interstice while the larger $\mathrm{Ne}, \mathrm{Ar}$ and $\mathrm{Kr}$ atoms all prefer octahedral interstice in group 5B metals, we plot in Fig. 5 the formation energies of $\mathrm{He}$ and $\mathrm{Ne}$ at both tetrahedral and octahedral sites in bcc $\mathrm{Nb}$ as a function of the degree of local relaxations measured by $x$. As has already been discussed, in unrelaxed calculations $(x=0)$, both He and Ne strongly prefer the tetrahedral site. However, the energy gain upon local relaxation for $\mathrm{Ne}$ in an octahedral site $(9.99 \mathrm{eV})$ is significantly larger than that in a tetrahedral site $(6.82 \mathrm{eV})$. This large difference in relaxation energy is sufficient to incur a site 
preference reversal, making octahedral the energetically more favorable site for Ne. Due to its smaller size, however, the relaxation energies for He occupying tetrahedral and octahedral sites are only 0.84 and $1.28 \mathrm{eV}$, respectively. In this case, while relaxation remains stronger at the octahedral site, the small difference in relaxation energy is not enough to reverse the unrelaxed site preference that favors the tetrahedral site.

\subsection{Theoretical model based on effective-medium theory}

Assuming isotropic elasticity, the elastic contribution in Eq. (4) can be estimated as the elastic energy cost associated with the insertion of an incompressible spherical inclusion (i.e., with zero compressibility) with radius $r=r_{0}+x$ into a spherical hole of original radius $r_{0}$ in a homogenous medium: ${ }^{25}$

$$
E_{\text {elastic }}(x)=8 \pi G x^{2} r_{0}
$$

where $G$ is the elastic shear modulus of the host bcc metal that can be obtained from experimental measurements. ${ }^{26}$ The radius $r_{0}$ is associated with the free volume of an interstitial site and its value can be fitted to DFT calculated elastic energies.

According to the effective-medium theory (EMT), ${ }^{18-20}$ the electronic energy can be further approximated as:

$$
E_{\text {electronic }}(x)=\alpha_{\text {eff }} \rho(x)
$$

where the proportionality constant $\alpha_{\text {eff }}$ measures the strength of repulsive interaction between a noble gas atom and host metal electron density. $\rho(x)$ is the local electron charge density at the 
center of an interstitial site after local relaxation of metal atoms. Assuming exponential decay of atomic charge densities, $\rho(x)$ can be written as:

$$
\rho(x)=\rho_{0} e^{-x / b}
$$

Here $\rho_{0}$ is the interstitial charge density in a perfect metal lattice while $b$ controls how fast the atomic charge density decays. It is worth noting that $r_{0}, \rho_{0}$ and $b$ depend only on the host metal and type of interstitial site, while $\alpha_{\text {eff }}$ only depends on the type of gas atom according to effective-medium theory.

A minimization of Eq. (4) with respect to $x$ gives the fully relaxed formation energy as:

$$
E_{\text {int }}^{f}=\alpha_{\text {eff }} \rho_{0} W(u)(2+W(u)) / 2 u
$$

where $W(u)$ is the Lambert-W function defined as the inverse function of $f(W)=W e^{W}$. The parameter $u$ measures the relative strength of electronic and elastic contributions to the formation energy and is given by:

$$
u=\alpha_{\text {eff }} \rho_{0} / 16 \pi G r_{0} b^{2}
$$

For realistic values of $u(0.5<u<13)$, Eq. (8) can be further approximated with reasonable accuracy as:

$$
E_{\text {int }}^{f}=\alpha_{e f f} \rho_{0}(0.7222-0.1719 \ln (u))
$$


Evidently, the $u$ parameter directly determines the ratio of relaxed to unrelaxed formation energy of a noble gas atom. Greater $u$ value will lead to larger relaxation energy and consequently smaller relaxed formation energy, and vice versa. Since $u$ is inversely proportional to the shear modulus $G$ of the host metal, it can be inferred from Eq. (10) that the fully relaxed formation energy of a noble gas atom should increase with increasing shear modulus of the bcc metal. This can be understood since a large shear modulus will thwart the energy-reducing local deformation process. In fact, for a rigid metal lattice with infinitely large $G, u$ will be close to zero. In this extreme case, the Lambert-W function $W(u)$ will asymptotically approach $u$ and $E_{\text {int }}^{f}$ will approach its unrelaxed value, $\alpha_{e f f} \rho_{0}$.

To substantiate this conclusion, we plot in Fig. 6 the fully relaxed formation energies of noble gas atoms against the polycrystalline shear moduli of the bcc metals, calculated using the Voigt approach as:

$$
G_{V}=\left(c_{11}-c_{12}+3 c_{44}\right) / 5
$$

where the single crystal elastic constants $c_{i j}$ 's of the bcc metals (see Table 2) are obtained from experimental measurements. ${ }^{26}$ Remarkably, a strong correlation between $E_{\text {int }}^{f}$ and $G$ is clearly revealed in Fig. 6. In comparison, the correlation between $E_{\text {int }}^{f}$ and $\rho_{0}$ is much weaker (see Fig. 7). This indicates that the energetics of noble gas atoms in bcc transition metals are more strongly governed by the local relaxation process rather than the initial electronic interactions between the gas atom and the perfect metal lattice. Due to the filling of $d$ states with strong directional bonding character, ${ }^{27} \mathrm{~W}$ exhibits the largest elastic shear modulus among all bcc 
transition metals, which explains why the fully relaxed formation energies of noble gas atoms have their highest values in this metal. Without allowing for local relaxations, however, the unrelaxed formation energies of noble gas interstitials in $\mathrm{W}$ can actually be smaller than those in $\mathrm{Cr}$ (see Fig. 2), which is due to the higher interstitial charge density in the latter.

To test the predicting power of our model, we have parameterized $r_{0}, \rho_{0}, b$ and $\alpha_{e f f}$ using DFT calculations for the case of noble gas atoms occupying tetrahedral sites in bcc transition metals. Our fitted values are reported in Tables 2 and 3. DFT calculated electronic embedding energies, elastic energies, and interstitial charge densities are shown in Figs. 8, 9 and 10, respectively. With the exception of $\mathrm{Nb}, r_{0}$ for the tetrahedral site in bcc transition metals is around $1.07 \AA$, which is comparable to the value for the tetrahedral site in $\operatorname{Si}(1.08 \AA) .{ }^{25}$ The parameter $b$ is around $0.38 \AA$ for the investigated bcc metals. As shown in Fig. 11, the model predicted formation energies of noble gas interstitials are in quantitative agreement with DFT results with a root-mean-square deviation (RMSD) of $0.53 \mathrm{eV}$. We attribute this discrepancy to the approximation made in the effective-medium theory, i.e., the embedding energy of a noble gas impurity in a metal lattice is proportional to the host charge density.

\subsection{Ground-state lattice configurations and migration energies}

Under irradiations, gas bubbles grow by capturing incoming gas atoms and vacancies. To predict the diffusivities of noble gas atoms in bcc transition metals, knowledge of their ground state (hence thermodynamically the most probable) lattice configurations is necessary. In addition to the tetrahedral and octahedral positions, we have also included trigonal (Fig. 1(c)) and crowdion (Fig. 1(d)) sites in our calculations, which have three and two nearest-neighbor metal atoms, 
respectively. Furthermore, following our previously proposed closed-system concept, ${ }^{28}$ we have considered the situation when an interstitial gas atom becomes substitutional by pushing a nearby metal atom away from its normal lattice position to form an isolated self-interstitial atom (SIA), which is located at first-nearest-neighbor (1NN) distance from the gas atom. The corresponding reaction $X_{\text {int }} \rightarrow X_{\text {sub }}+$ SIA is similar to the self-trapping process, ${ }^{29}$ but involves only a single gas atom. It is also the reverse of the replacement reaction, ${ }^{6}$ i.e., a SIA spontaneously recombines with the vacancy occupied by a substitutional gas atom and kicks the gas atom back to an interstitial position. Possible SIA configurations include $<111>$ and $<110>$ dumbbells in different orientations relative to the gas atom. We exclude $<100>$ dumbbells from our consideration in view of their consistently higher energies in bcc metals. ${ }^{24,30}$ All these configurations are illustrated in Fig. 1. In most cases, a noble gas atom is most stable at either a tetrahedral or an octahedral interstitial site. For $\mathrm{Kr}$ in $\mathrm{Cr}$, however, the trigonal site is $0.31 \mathrm{eV}$ more stable than tetrahedral site. In the case of $\mathrm{Ar}$ in $\mathrm{Fe}$, the trigonal site is also $0.05 \mathrm{eV}$ lower in energy than the tetrahedral site. Remarkably, we find that a single $\mathrm{Kr}$ interstitial is not energetically stable in Fe and will relax into a substitutional position by punching out a $<110>$ dumbbell SIA, followed by a total energy reduction of $0.41 \mathrm{eV}$. The final ground state configuration for $\mathrm{Kr}$ in Fe is shown in Fig. 1(h). Using the method described in Ref. 11, we determine the binding energy between the SIA and substitutional $\mathrm{Kr}$ to be only $0.12 \mathrm{eV}$, indicating a rather weak binding between the two defects. By adding the migration energy of $<110>$ dumbbell in bcc $\mathrm{Fe}\left(0.34 \mathrm{eV}^{30}\right)$, the corresponding dissociation energy is $0.46 \mathrm{eV}$. Once dissociated, the freely migrating SIA can either get annihilated at defect sinks such as grain 
boundaries or free surfaces, or aggregate with each other to form larger defect clusters such as interstitial Frank loops.

For gas atoms that prefer the octahedral sites, the minimum energy path (MEP) for migration is between neighboring octahedral sites along the $<100>$ direction, passing through a tetrahedral site as the saddle point. ${ }^{7}$ The migration barrier for gas atom diffusion can be calculated from the energy difference between the octahedral and the tetrahedral site as $E_{O c t-O c t}^{m i g}=E_{\text {Tet }}^{f}-E_{O c t}^{f}$. When tetrahedral sites are the most stable, the MEP for gas atom hopping is between adjacent tetrahedral sites along the $<110>$ direction, with a trigonal site being the transition state. ${ }^{14}$ The corresponding migration barrier is therefore $E_{T e t-T e t}^{m i g}=E_{T r i}^{f}-E_{T e t}^{f}$. While it is also possible for gas atoms to jump from one tetrahedral site to another through an octahedral site along the $<100>$ direction, this diffusion path has much higher migration barrier and will not be the dominant one. For gas atoms that prefer trigonal sites, we calculate the migration barrier for interstitial gas atom hopping between neighboring trigonal sites using a climbing image nudged elastic band (CI-NEB) technique ${ }^{31}$ with three intermediate images in a 128 -atom $4 \times 4 \times 4$ cubic bcc supercell. Finally, for interstitial gas atoms that spontaneously relax into substitutional positions, their effective migration barriers are not well defined and strongly depend on whether vacancies are at thermal equilibrium or are superabundant due to irradiation. ${ }^{9}$ Our final calculated migration barriers are reported in Table 1, in excellent agreement with previous studies. ${ }^{9,11,14-16}$ It is noteworthy that, while interstitial $\mathrm{He}$ and Ne atoms can migrate in all bcc transition metals with relatively low barriers, the migration energies for interstitial $\mathrm{Ar}$ and $\mathrm{Kr}$ atoms are high in group 5B metals, where octahedral is the preferred site. 


\subsection{Interactions with irradiation-induced vacancies}

Our discussions so far assume inert gas atoms interacting with a pristine bcc lattice with no preexisting defects. Under thermodynamic equilibrium conditions, the concentrations of thermal point defects in bcc transition metals can indeed be negligibly low due to their high formation energies. ${ }^{24}$ Under irradiation, however, excess vacancies and SIAs will be generated in amounts significantly greater than their equilibrium concentrations. Using Eq. (3), we have calculated the binding energies between noble gas interstitials and vacancies in bcc transition metals. Our results are shown in Table 1 , in good agreement with previous studies..$^{9,12,13,15,16}$ In all cases, vacancies can strongly trap interstitial gas atoms and serve as the nuclei for gas bubble formation, with gas-vacancy binding being particularly strong for group 6B metals and large noble gas atoms. Since a $\mathrm{Kr}$ interstitial is not stable in bcc Fe and will spontaneously punch out a self interstitial atom and becomes immobile substitutional, the gas-vacancy binding energy in this case is actually the formation energy of a Frenkel pair in bec Fe $(6.23 \mathrm{eV})$ minus the binding energy between the punched-out SIA and substitutional $\mathrm{Kr}(0.12 \mathrm{eV})$. Furthermore, only for He substitutional in bcc V, we find that moving He off vacancy center along [100] and [110] directions will reduce the total energy by 0.22 and $0.19 \mathrm{eV}$, respectively, which is in agreement with Seletskaia et al. ${ }^{12}$

When $E_{X_{\text {int }}-V a}^{\text {bind }}$ is significantly greater than $E_{V a}^{f}$, as is the case for $\mathrm{Ne}, \mathrm{Ar}$ and $\mathrm{Kr}$ in all bcc transition metals, the dissociation mechanism is unlikely to play a dominant role for the migration of substitutional gas atoms, and their diffusion would either need the assistance of an additional vacancy to form a transient and mobile gas-divacancy complex, ${ }^{9}$ or need an incoming 
SIA to force the gas atom back to an interstitial site via the replacement reaction. ${ }^{6}$ Conversely, for He in group 5B metals, the gas-vacancy binding energy is only about half of the vacancy formation energy, as was also found by Seletskaia et al. ${ }^{12}$ In this case, in addition to the vacancy and replacement mechanisms, the gas atoms trapped inside a vacancy can return to the metal lattice and rapidly migrate as an interstitial, until they are trapped by another defect.

\section{Conclusions}

In summary, extensive $a b$ initio calculations have been performed to predict the lattice site preference, formation energies, binding energies with vacancy, and migration paths and barriers of noble gas atoms in bec transition metals. Without allowing for local relaxations of the metal lattice, all four noble gas atoms energetically prefer tetrahedral over octahedral interstitial sites in all bce transition metals. This can be explained by the significant polarization of the two firstnearest-neighbor metal atoms of a noble gas interstitial at octahedral position due to the very short gas-metal distance. Remarkably, local relaxations of metal atoms can significantly reduce gas interstitial formation energies by as much as $75 \%$ at tetrahedral sites and $79 \%$ at octahedral sites, which is strong enough to reverse the tetrahedral vs. octahedral site preference for $\mathrm{Ne}, \mathrm{Ar}$ and $\mathrm{Kr}$ in group 5B metals. Unexpectedly, we find that the fully relaxed formation energies of noble gas interstitials are much more strongly correlated with the elastic shear moduli of bcc transition metals than with their charge densities. The large formation energies of noble gas interstitials in group 6B metals can therefore be alternatively explained by their exceptionally high elastic shear moduli driven by the development of directional $d-d$ bonding. Previously, this group-specific trend was attributed to the higher charge density values at interstitial region in 
group 6B metals by Seletskaia et al. ${ }^{12}$ in their study of $\mathrm{He}$ in bcc metals. In general, however, both interstitial charge density and elastic shear modulus are important parameters that need to be considered in quantitative modeling of the energetics of noble gas atoms in materials (e.g. Eq. (8)). In addition to presenting new physical understanding of gas-metal interactions, this study provides key inputs to kinetic Monte Carlo simulations of gas bubble nucleation and growth. ${ }^{32}$

\section{Conflicts of interest}

There are no conflicts to declare.

\section{Acknowledgements}

This work was fully sponsored by the U.S. Department of Energy, Office of Science, Basic Energy \& Science (BES), Materials Sciences and Engineering Division under FWP \#C000-14003 at Idaho National Laboratory operated by Battelle Energy Alliance (BEA) under DOE-NE Idaho Operations Office Contract DE-AC07-05ID14517. All DFT calculations are performed using the FALCON supercomputer at Idaho National Laboratory.

\section{References}

1 X. Dong, A. R. Oganov, A. F. Goncharov et al., Nat. Chem., 2017, 9, 440.

2 P. B. Johnson, R. W. Thomson and D. J. Mazey, Nature, 1990, 347, 265.

3 P. B. Johnson and D. J. Mazey, J. Nucl. Mater., 1995, 218, 273.

4 J. Gan, D. D. Keiser Jr., D. M. Wachs, A. B. Robinson, B. D. Miller and T. R. Allen, J. Nucl. Mater., 2010, 396, 234.

5 J. Gan, D. D. Keiser Jr., B. D. Miller, A. B. Robinson, J. F. Jue, P. Medvedev and D. M. Wachs, J. Nucl. Mater., 2012, 424, 43.

6 D. M. Stewart, Y. Osetsky, R. E. Stoller, S. I. Golubov, T. Seletskaia and P. J. Kamenski, 
Phil. Mag., 2010, 90, 935.

Y. F. Zhang, P. C. Millett and M. Tonks, Comput. Mater. Sci., 2011, 50, 3224.

L. Sandoval, D. Perez, B. P. Uberuaga and A. F. Voter, Phys. Rev. Lett., 2015, 114, 105502.

C. C. Fu and F. Willaime, Phys. Rev. B, 2005, 72, 064117.

T. Seletskaia, Y. Osetsky, R. E. Stoller and G. M. Stocks, Phys. Rev. Lett., 2005, 94, 046403.

C. Becquart and C. Domain, Phys. Rev. Lett., 2006, 97, 196402.

T. Seletskaia, Y. Osetsky, R. E. Stoller and G. M. Stocks, Phys. Rev. B, 2008, 78, 134103.

X. T. Zhu, L. Yang, F. Gao, S. M. Peng, H. L. Heinisch, X. G. Long and R. J. Kurtz, Phys. Rev. B, 2009, 80, 054104.

T. Tamura, R. Kobayashi, S. Ogata and A. Ito, Modelling Simul. Mater. Sci. Eng., 2014, 22, 015002 .

G. Y. Huang, N. Juslin and B. D. Wirth, Comput. Mater. Sci., 2016, 123, 121.

D. Nguyen-Manh and S. L. Dudarev, Nucl. Instrum. Meth. Phys. Res. B, 2015, 352, 86.

R. L. Klueh and A. T. Nelson, J. Nucl. Mater., 2007, 371, 37.

N. Esbjerg and J. K. Norskov, Phys. Rev. Lett., 1980, 45, 807.

M. Manninen, J. K. Norskov, M. J. Puska and C. Umrigar, Phys. Rev. B, 1984, 29, 2314.

B. B. Nielsen and A. van Veen, J. Phys. F:Met. Phys., 1985, 15, 2409.

J. P. Perdew, K. Burke and M. Ernzerhof, Phys. Rev. Lett., 1996, 77, 3865.

G. Kresse and J. Furthmuller, Phys. Rev. B, 1996, 54, 11169.

G. Kresse and D. Jouber, Phys. Rev. B, 1999, 59, 1758.

D. Nguyen-Manh, A. P. Horsfield and S. L. Dudarev, Phys. Rev. B, 2006, 73, 020102.

L. Pizzagalli, A. Charaf-Eddin and S. Brochard, Compu. Mater. Sci., 2014, 95, 149.

G. Simmons and H. Wang, Single Crystal Elastic Constants and Calculated Aggregate Properties, MIT press, Cambridge, 1971.

K. M. Ho, C. L. Fu and B. N. Harmon, Phys. Rev. B, 1983, 28, 6687.

C. Jiang, X. Y. Liu and K. Sickafus, Phys. Rev. B, 2011, 83, 052103. 
W. D. Wilson, C. L. Bisson and M. I. Baskes, Phys. Rev. B, 1981, 24, 5616.

C. C. Fu, F. Willaime, and P. Ordejon, Phys. Rev. Lett., 2004, 92, 175503.

G. Henkelman, B. P. Uberuaga, and H. Jonsson, J. Chem. Phys., 2000, 113, 9901. 
Table 1. DFT calculated formation energies $(\mathrm{eV})$, gas-vacancy binding energies $(\mathrm{eV})$ and migration barriers $(\mathrm{eV})$ for noble gas atoms in bcc transition metals, in comparison with previous DFT studies. Results shown in parentheses are from our unrelaxed DFT calculations.

\begin{tabular}{|c|c|c|c|c|c|}
\hline $\begin{array}{l}\text { Gas } \\
\text { atom }\end{array}$ & $\begin{array}{l}\text { Metal } \\
\text { atom }\end{array}$ & $E_{\text {Tet }}^{f}$ & $E_{\text {Oct }}^{f}$ & $E_{X_{\text {int }}-V a}^{\text {bind }}$ & $E^{m i g}$ \\
\hline \multirow{7}{*}{$\mathrm{He}$} & $\mathrm{V}$ & $3.17(4.19)^{\mathrm{a}}, 2.94^{\mathrm{e}}$ & $3.40(4.92)^{\mathrm{a}}, 3.17^{\mathrm{e}}$ & $1.16^{\mathrm{a}}, 0.91^{\mathrm{e}}$ & $0.09^{\mathrm{a}}$ \\
\hline & $\mathrm{Nb}$ & $3.13(4.04)^{\mathrm{a}}, 3.05^{\mathrm{e}}$ & $3.40(4.72)^{\mathrm{a}}, 3.26^{\mathrm{e}}$ & $1.49^{\mathrm{a}}, 1.67^{\mathrm{e}}$ & $0.09^{\mathrm{a}}$ \\
\hline & $\mathrm{Ta}$ & $3.46(4.38)^{\mathrm{a}}, 3.16^{\mathrm{e}}$ & $3.79(5.05)^{\mathrm{a}}, 3.42^{\mathrm{e}}$ & $1.79^{\mathrm{a}}, 1.41^{\mathrm{e}}$ & $0.09^{\mathrm{a}}$ \\
\hline & $\mathrm{Cr}$ & $5.22(6.50)^{\mathrm{a}}, 5.22^{\mathrm{f}}$ & $5.40(7.17)^{\mathrm{a}}, 5.38^{\mathrm{f}}$ & $2.84^{\mathrm{a}}, 2.83^{\mathrm{f}}$ & $0.08^{\mathrm{a}}$ \\
\hline & Mo & $\begin{array}{c}5.31(6.51)^{\mathrm{a}}, 5.16^{\mathrm{e}} \\
5.33^{\mathrm{f}}\end{array}$ & $\begin{array}{c}5.49(6.94)^{\mathrm{a}}, 5.33^{\mathrm{e}}, \\
5.48^{\mathrm{f}}\end{array}$ & $3.65^{\mathrm{a}}, 3.40^{\mathrm{e}}, 3.66^{\mathrm{f}}$ & $0.06^{\mathrm{a}}$ \\
\hline & W & $\begin{array}{c}6.31(7.54)^{\mathrm{a}}, 6.16^{\mathrm{d}}, \\
6.15^{\mathrm{e}}, 6.19^{\mathrm{f}}, 6.29^{\mathrm{g}} \\
\quad 6.23^{\mathrm{h}}, 6.22^{\mathrm{i}}\end{array}$ & $\begin{array}{c}6.52(7.93)^{\mathrm{a}}, 6.38^{\mathrm{d}}, \\
6.39^{\mathrm{e}}, 6.41^{\mathrm{f}}, 6.67^{\mathrm{g}}, \\
\quad 6.48^{\mathrm{h}}, 6.44^{\mathrm{i}}\end{array}$ & $\begin{array}{c}4.75^{\mathrm{a}}, 4.77^{\mathrm{e}}, 4.65^{\mathrm{f}} \\
4.62^{\mathrm{h}}, 4.63^{\mathrm{i}}\end{array}$ & $\begin{array}{c}0.06^{\mathrm{a}}, 0.06^{\mathrm{d}}, 0.06^{\mathrm{g}} \\
0.07^{\mathrm{h}}, 0.07^{\mathrm{i}}\end{array}$ \\
\hline & $\mathrm{Fe}$ & $\begin{array}{c}4.55(5.76)^{\mathrm{a}}, 4.39^{\mathrm{b}} \\
4.37^{\mathrm{c}}, 4.56^{\mathrm{f}}\end{array}$ & $\begin{array}{c}4.75(6.30)^{\mathrm{a}}, 4.58^{\mathrm{b}}, \\
\quad 4.60^{\mathrm{c}}, 4.75^{\mathrm{f}}\end{array}$ & $2.34^{\mathrm{a}}, 2.30^{\mathrm{b}}, 2.36^{\mathrm{f}}$ & $0.06^{\mathrm{a}}, 0.06^{\mathrm{b}}$ \\
\hline \multirow{7}{*}{$\mathrm{Ne}$} & V & $6.56(14.52)^{\mathrm{a}}$ & $6.39(17.63)^{\mathrm{a}}$ & $3.36^{\mathrm{a}}$ & $0.17^{\mathrm{a}}$ \\
\hline & $\mathrm{Nb}$ & $6.24(13.26)^{\mathrm{a}}$ & $6.17(16.17)^{\mathrm{a}}$ & $3.92^{\mathrm{a}}$ & $0.07^{\mathrm{a}}$ \\
\hline & $\mathrm{Ta}$ & $7.04(13.87)^{\mathrm{a}}$ & $6.98(16.83)^{\mathrm{a}}$ & $4.52^{\mathrm{a}}$ & $0.06^{\mathrm{a}}$ \\
\hline & $\mathrm{Cr}$ & $10.10(20.14)^{\mathrm{a}}$ & $10.34(23.59)^{\mathrm{a}}$ & $6.04^{\mathrm{a}}$ & $0.12^{\mathrm{a}}$ \\
\hline & Mo & $9.61(18.56)^{\mathrm{a}}$ & $9.94(21.28)^{\mathrm{a}}$ & $6.82^{\mathrm{a}}$ & $0.13^{\mathrm{a}}$ \\
\hline & W & $\begin{array}{l}11.41(20.32)^{\mathrm{a}}, \\
11.55^{\mathrm{g}}, 11.40^{\mathrm{h}}\end{array}$ & $\begin{array}{l}11.89(22.93)^{\mathrm{a}}, \\
12.17^{\mathrm{g}}, 11.95^{\mathrm{h}}\end{array}$ & $8.30^{\mathrm{a}}, 8.23^{\mathrm{h}}, 8.18^{\mathrm{i}}$ & $\begin{array}{c}0.14^{\mathrm{a}}, 0.17^{\mathrm{g}}, 0.15^{\mathrm{h}}, \\
0.14^{\mathrm{i}}\end{array}$ \\
\hline & $\mathrm{Fe}$ & $8.93(19.17)^{\mathrm{a}}$ & $9.03(21.77)^{\mathrm{a}}$ & $5.36^{\mathrm{a}}$ & $0.02^{\mathrm{a}}$ \\
\hline \multirow{7}{*}{$\mathrm{Ar}$} & V & $8.42(22.45)^{\mathrm{a}}$ & $7.94(25.93)^{\mathrm{a}}$ & $3.33^{\mathrm{a}}$ & $0.48^{\mathrm{a}}$ \\
\hline & $\mathrm{Nb}$ & $8.35(20.23)^{\mathrm{a}}$ & $7.80(24.69)^{\mathrm{a}}$ & $3.97^{\mathrm{a}}$ & $0.54^{\mathrm{a}}$ \\
\hline & $\mathrm{Ta}$ & $9.34(20.94)^{\mathrm{a}}$ & $8.78(25.21)^{\mathrm{a}}$ & $4.51^{\mathrm{a}}$ & $0.56^{\mathrm{a}}$ \\
\hline & $\mathrm{Cr}$ & $12.85(29.92)^{\mathrm{a}}$ & $13.36(34.45)^{\mathrm{a}}$ & $6.34^{\mathrm{a}}$ & $0.05^{\mathrm{a}}$ \\
\hline & Mo & $12.71(27.07)^{\mathrm{a}}$ & $13.39(32.74)^{\mathrm{a}}$ & $7.36^{\mathrm{a}}$ & $0.20^{\mathrm{a}}$ \\
\hline & W & $\begin{array}{l}14.92(29.71)^{\mathrm{a}}, \\
14.99^{\mathrm{g}}, 15.10^{\mathrm{h}}\end{array}$ & $\begin{array}{l}16.06(35.84)^{\mathrm{a}}, \\
16.05^{\mathrm{g}}, 16.29^{\mathrm{h}}\end{array}$ & $8.75^{\mathrm{a}}, 8.82^{\mathrm{h}}, 8.54^{\mathrm{i}}$ & $\begin{array}{c}0.25^{\mathrm{a}}, 0.19^{\mathrm{g}}, 0.27^{\mathrm{h}} \\
0.23^{\mathrm{i}}\end{array}$ \\
\hline & $\mathrm{Fe}$ & $11.50(26.21)^{\mathrm{a}}$ & $11.63(31.08)^{\mathrm{a}}$ & $5.72^{\mathrm{a}}$ & $0.03^{\mathrm{a}}$ \\
\hline \multirow{7}{*}{$\mathrm{Kr}$} & $\mathrm{V}$ & $9.32(37.29)^{\mathrm{a}}$ & $9.12(42.76)^{a}$ & $4.56^{\mathrm{a}}$ & $0.21^{\mathrm{a}}$ \\
\hline & $\mathrm{Nb}$ & $9.20(32.23)^{\mathrm{a}}$ & $8.76(38.60)^{\mathrm{a}}$ & $4.90^{\mathrm{a}}$ & $0.45^{\mathrm{a}}$ \\
\hline & $\mathrm{Ta}$ & $10.45(33.48)^{\mathrm{a}}$ & $9.97(39.55)^{\mathrm{a}}$ & $5.65^{\mathrm{a}}$ & $0.47^{\mathrm{a}}$ \\
\hline & $\mathrm{Cr}$ & $14.69(49.72)^{\mathrm{a}}$ & $15.35(57.33)^{\mathrm{a}}$ & $7.62^{\mathrm{a}}$ & $0.27^{\mathrm{a}}$ \\
\hline & Mo & $14.36(43.04)^{\mathrm{a}}$ & $15.08(51.25)^{\mathrm{a}}$ & $8.64^{\mathrm{a}}$ & $0.02^{\mathrm{a}}$ \\
\hline & $\mathrm{W}$ & $16.97(46.76)^{\mathrm{a}}$ & $18.16(55.32)^{\mathrm{a}}$ & $10.40^{\mathrm{a}}$ & $0.03^{\mathrm{a}}$ \\
\hline & $\mathrm{Fe}$ & $12.73(42.65)^{\mathrm{a}}$ & $13.03(49.98)^{\mathrm{a}}$ & $6.12^{\mathrm{a}}$ & \\
\hline
\end{tabular}

"ahis work

${ }^{\mathrm{b}}$ Reference 9

${ }^{\mathrm{c}}$ Reference 10

${ }^{\mathrm{d}}$ Reference 11

${ }^{\mathrm{e}}$ Reference 12

${ }^{f}$ Reference 13

${ }^{g}$ Reference 14

${ }^{\mathrm{h}}$ Reference 15

${ }^{\mathrm{i}}$ Reference 16 
Table 2. Values of $r_{0}, \rho_{0}$ and $b$ for tetrahedral interstitial sites in bcc transition metals obtained from the present DFT calculations. The single crystal elastic constants $c_{i j}$ 's from Ref. 26 and the calculated polycrystalline shear moduli of pure bcc elements are also shown.

\begin{tabular}{cccccccc}
\hline \hline Bcc metal & $r_{0}(\AA)$ & $b(\AA)$ & $\rho_{0}\left(\mathrm{e} / \AA^{3}\right)$ & $c_{11}(\mathrm{GPa})$ & $c_{12}(\mathrm{GPa})$ & $c_{44}(\mathrm{GPa})$ & $G_{V}(\mathrm{GPa})$ \\
\hline $\mathrm{V}$ & 1.129 & 0.397 & 0.242 & 232 & 119 & 46 & 50 \\
\hline $\mathrm{Nb}$ & 1.460 & 0.401 & 0.207 & 253 & 133 & 31 & 42 \\
\hline $\mathrm{Ta}$ & 1.066 & 0.378 & 0.227 & 266 & 158 & 87 & 74 \\
\hline $\mathrm{Cr}$ & 0.985 & 0.379 & 0.286 & 391 & 90 & 103 & 122 \\
\hline $\mathrm{Mo}$ & 1.050 & 0.373 & 0.249 & 473 & 156 & 111 & 130 \\
\hline $\mathrm{W}$ & 1.099 & 0.368 & 0.272 & 533 & 205 & 163 & 163 \\
\hline $\mathrm{Fe}$ & 1.095 & 0.371 & 0.249 & 243 & 138 & 122 & 94 \\
\hline \hline
\end{tabular}


Table 3. Values of $\alpha_{\text {eff }}$ for noble gas atoms obtained from the present DFT calculations.

\begin{tabular}{cc}
\hline \hline Gas atom & $\alpha_{\text {eff }}\left(\mathrm{eV} \AA^{3}\right)$ \\
\hline $\mathrm{He}$ & 28.69 \\
\hline $\mathrm{Ne}$ & 67.91 \\
\hline $\mathrm{Ar}$ & 101.57 \\
\hline $\mathrm{Kr}$ & 130.26 \\
\hline \hline
\end{tabular}




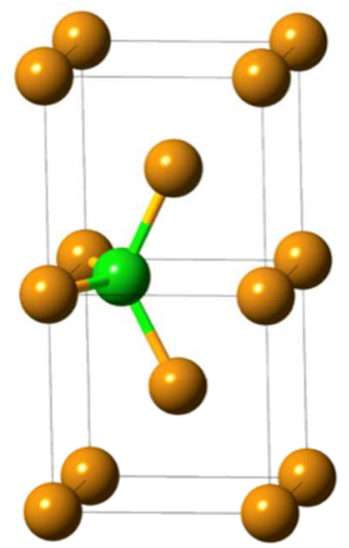

(a)

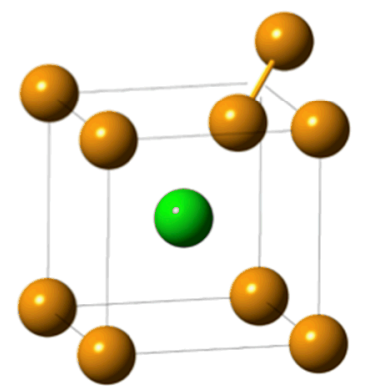

(e)

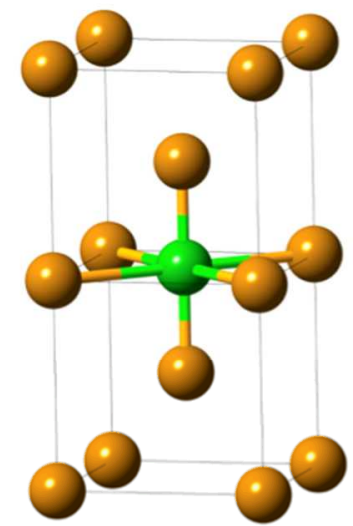

(b)

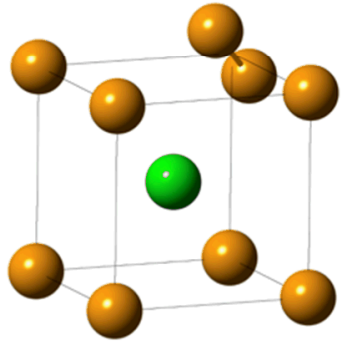

(f)

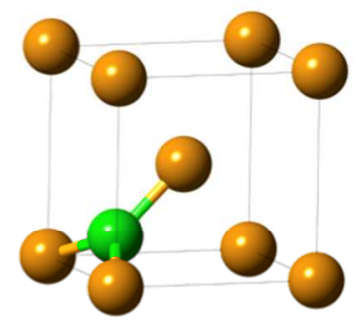

(c)

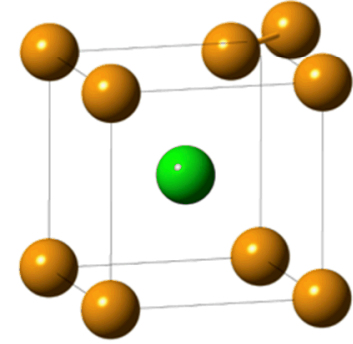

$(\mathrm{g})$

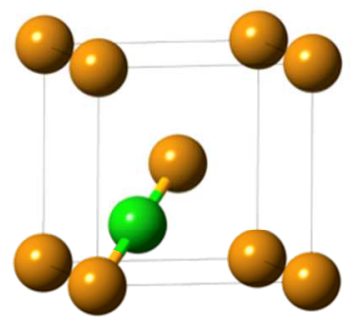

(d)

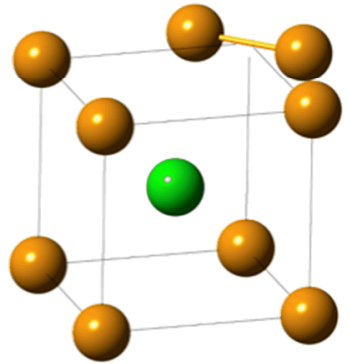

(h)

Fig. 1. Possible lattice configurations of a noble gas atom incorporated into a perfect bcc lattice:

(a) tetrahedral, (b) octahedral, (c) trigonal, (d) crowdion, (e) $\mathrm{X}_{\mathrm{sub}}+$ [111] dumbbell, (f) $\mathrm{X}_{\mathrm{sub}}+[11-1]$ dumbbell, (g) $\mathrm{X}_{\mathrm{sub}}+[110]$ dumbbell, and (h) $\mathrm{X}_{\text {sub }}+[1-10]$ dumbbell. The yellow and green spheres denote metal and gas atoms, respectively. 

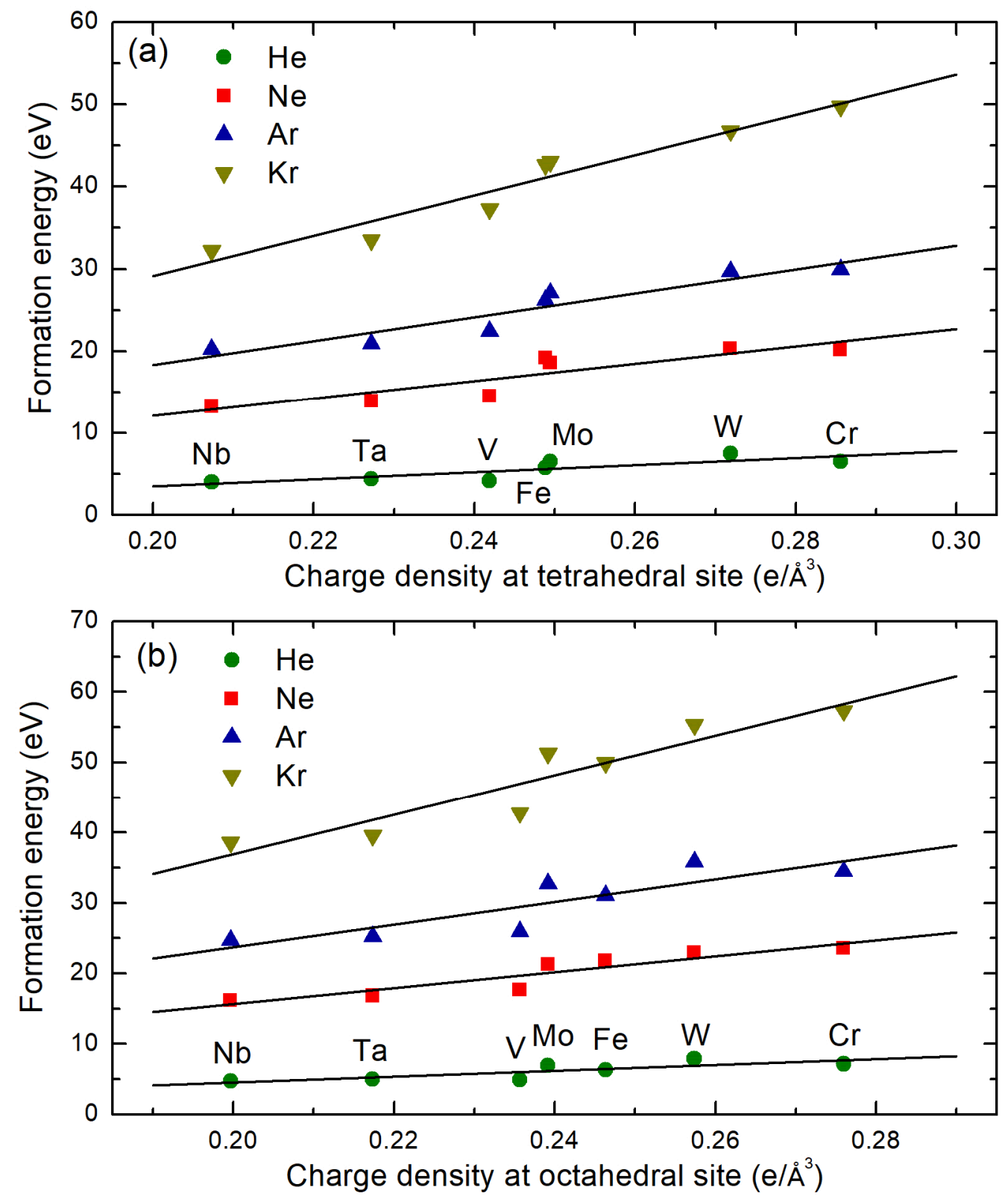

Fig. 2. Unrelaxed formation energies of noble gas atoms at (a) tetrahedral and (b) octahedral sites in bcc transition metals from the present DFT calculations, plotted as a function of the unperturbed interstitial charge density in the host bcc metal. The solid lines are guides to the eye. 

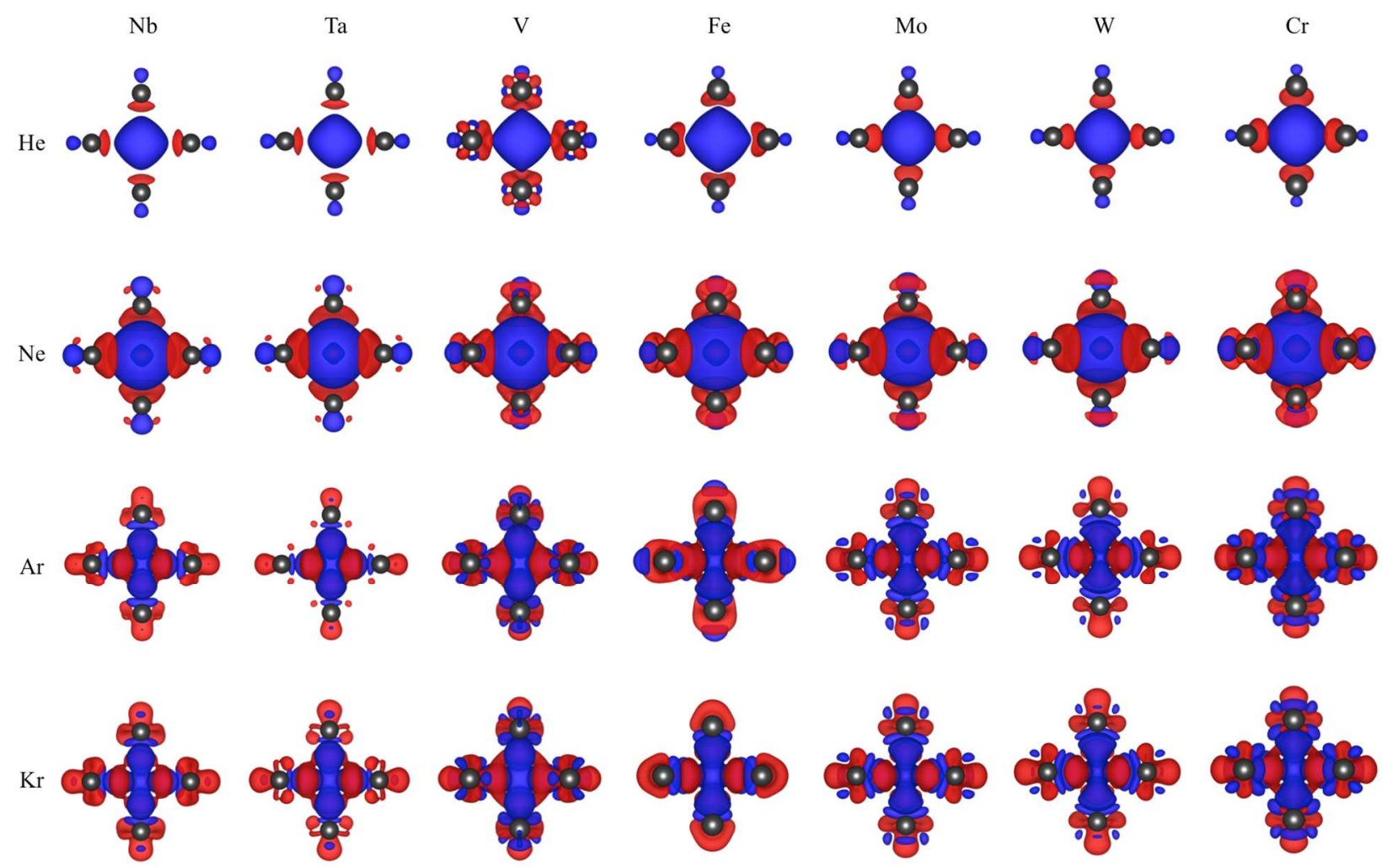

Fig. 3. The change in charge density in bcc transition metals after insertion of a noble gas atom at tetrahedral interstitial site. Isosurfaces at $\Delta \rho=+0.05 \mathrm{e} / \AA^{3}$ (red) and $-0.05 \mathrm{e} / \AA^{3}$ (blue) are shown. All metal atoms are at their ideal lattice positions. The order of bcc metals (from left to right) represents increasing charge density at the tetrahedral site. The order of noble gas atoms (from top to down) represents increasing size. The spheres give the positions of neighboring metal atoms. 

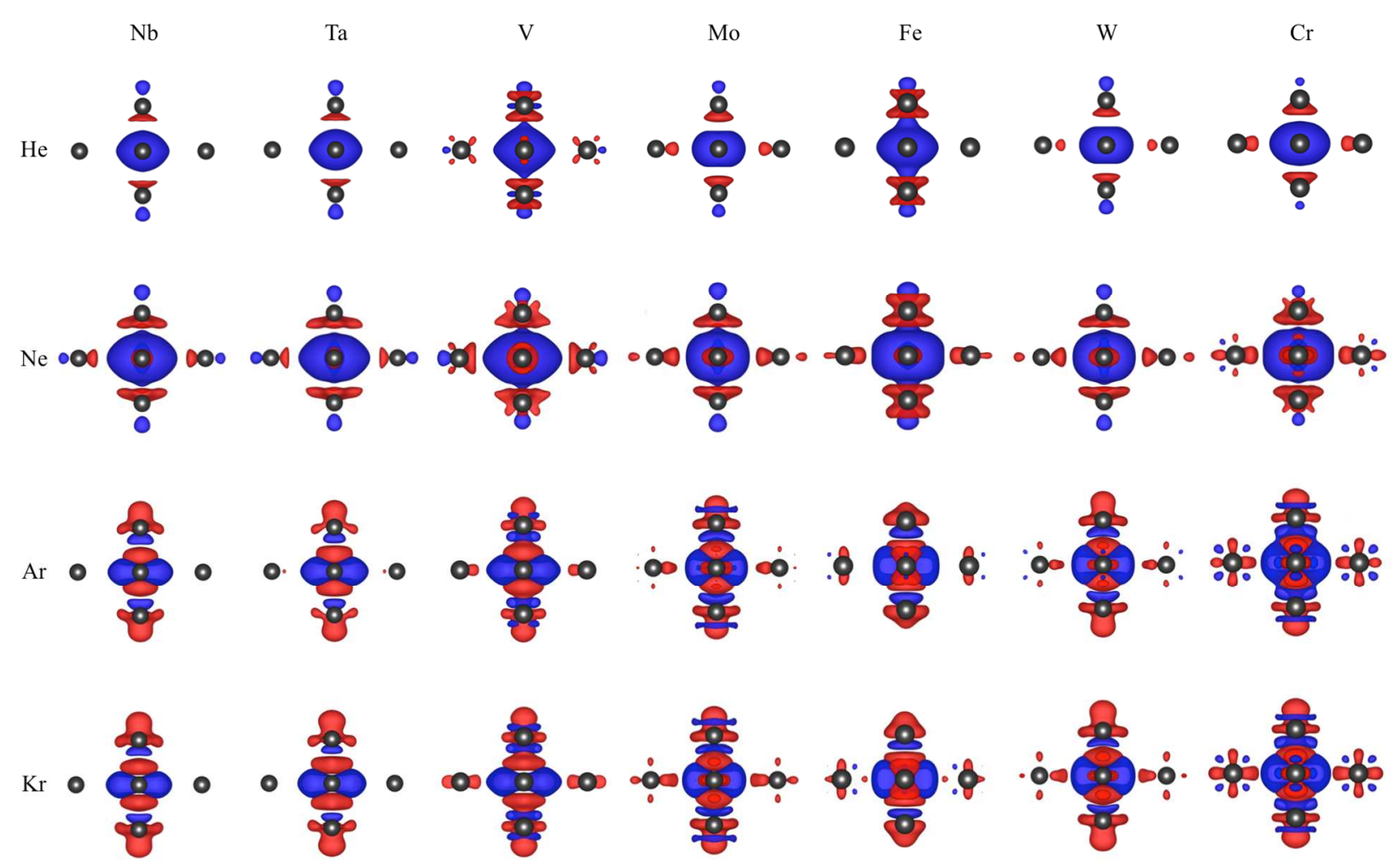

Fig. 4. The change in charge density in bec transition metals after insertion of a noble gas atom at octahedral interstitial site. Isosurfaces at $\Delta \rho=+0.05 \mathrm{e} / \AA^{3}$ (red) and $-0.05 \mathrm{e} / \AA^{3}$ (blue) are shown. All metal atoms are at their ideal lattice positions. The order of bcc metals (from left to right) represents increasing charge density at the octahedral site. The order of noble gas atoms (from top to down) represents increasing size. The spheres give the positions of neighboring metal atoms. 


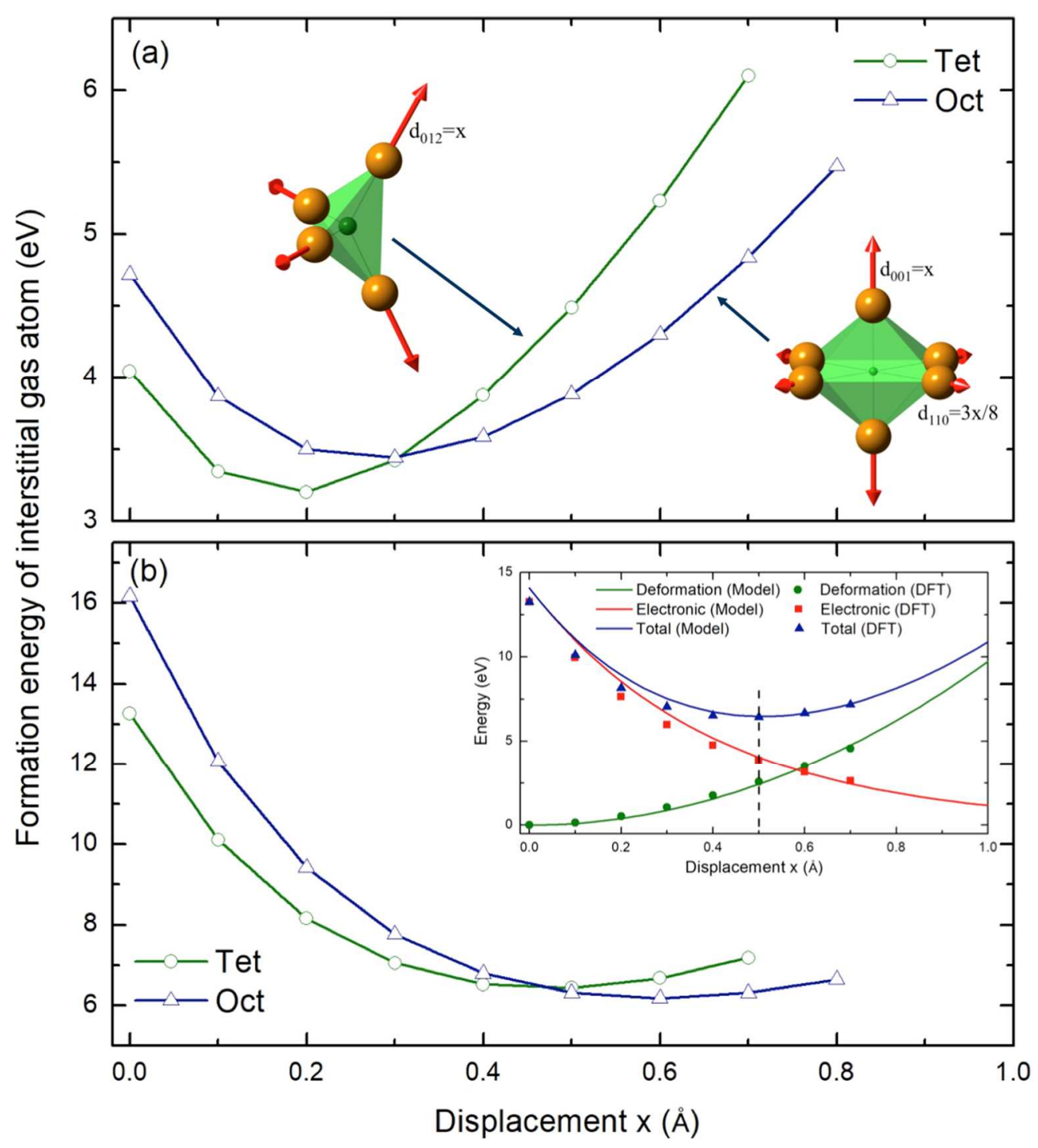

Fig. 5. DFT calculated formation energies of (a) He and (b) Ne occupying tetrahedral and octahedral sites in bcc $\mathrm{Nb}$, plotted as a function of $x$. The insets in (a) illustrate the local displacement of metal atoms surrounding a tetrahedral and an octahedral site in a bcc metal. The inset in (b) shows the decomposition of the total formation energy into deformation and electronic contributions for $\mathrm{Ne}$ at tetrahedral site in bcc $\mathrm{Nb}$. The vertical dashed line indicates the location of the minimum formation energy. 


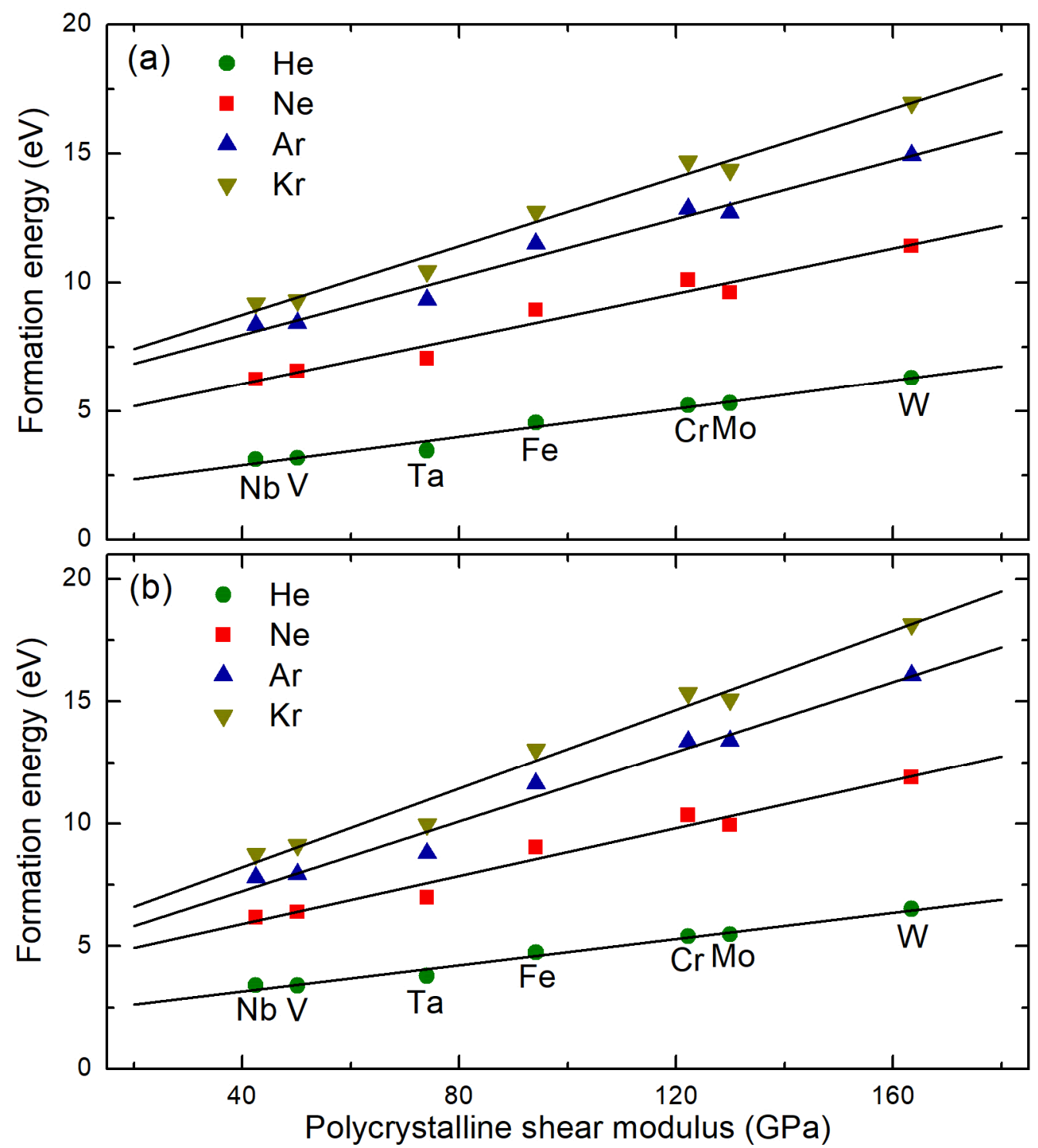

Fig. 6. Fully relaxed formation energies of noble gas atoms at (a) tetrahedral and (b) octahedral sites in bcc transition metals from the present DFT calculations, plotted against the polycrystalline shear modulus of the host bcc metal. The solid lines are guides to the eye. 

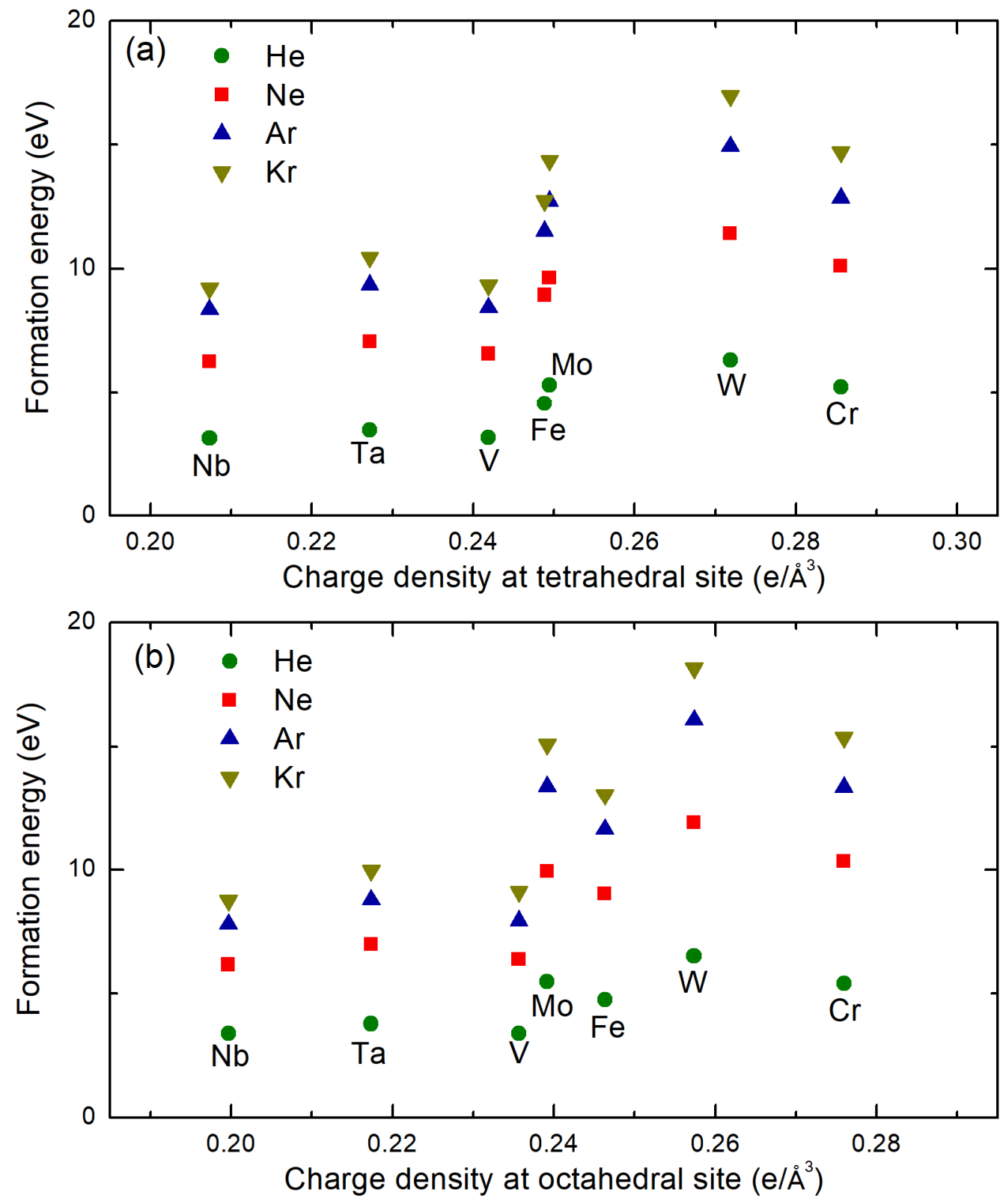

Fig. 7. Fully relaxed formation energies of noble gas atoms at (a) tetrahedral and (b) octahedral sites in bcc transition metals from the present DFT calculations, plotted as a function of the unperturbed interstitial charge density in the host bcc metal. 


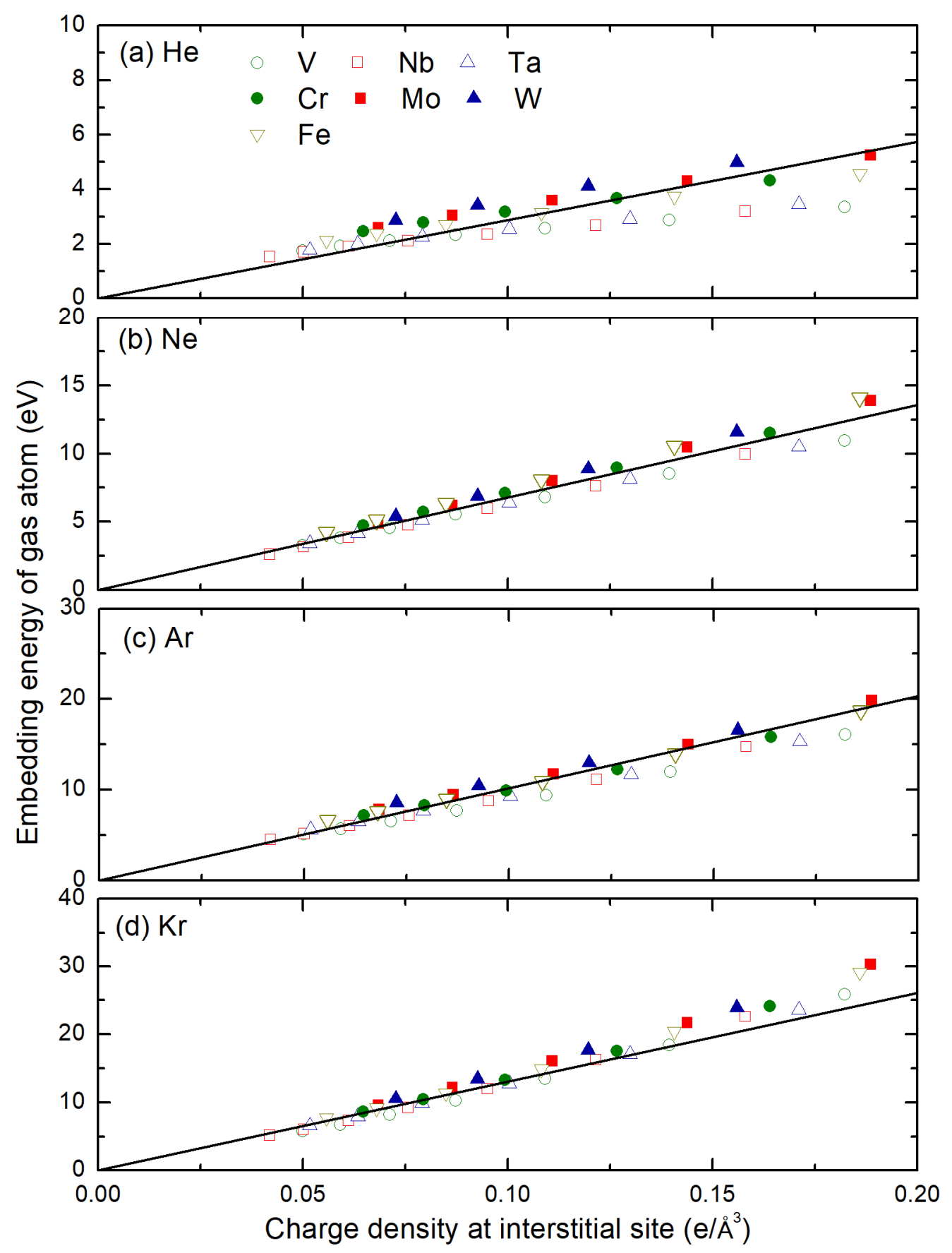

Fig. 8. DFT calculated electronic embedding energies of noble gas atoms at tetrahedral sites in bcc transition metals plotted as a function of the interstitial charge density from self-consistent DFT calculations. The solid lines represent the best linear fitting of DFT data using Eq. (6). 

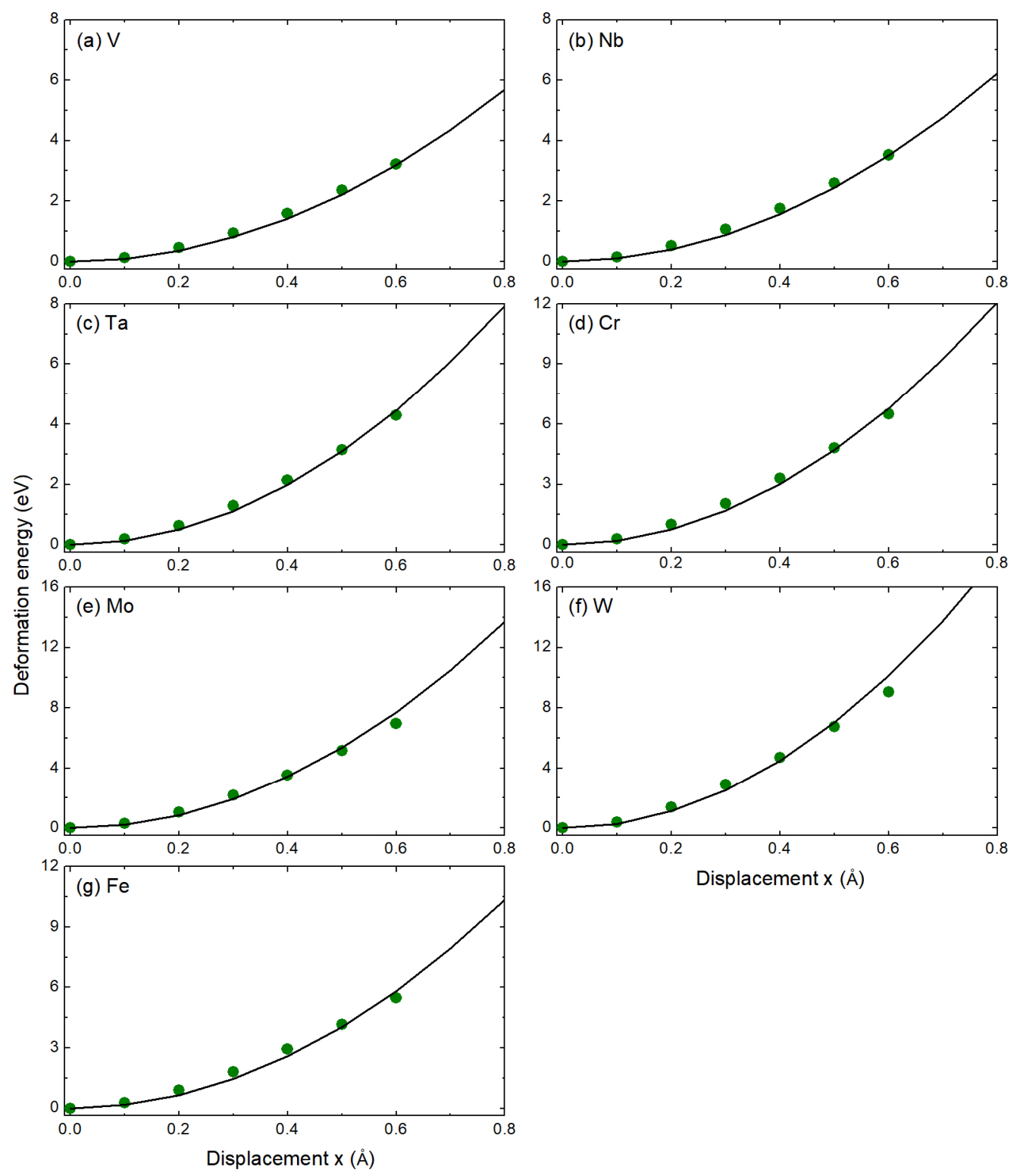

Fig. 9. DFT calculated elastic deformation energies for tetrahedral sites in bcc transition metals plotted as a function of the local displacement of metal atoms. The solid lines represent the best quadratic fitting of DFT data using Eq. (5). 

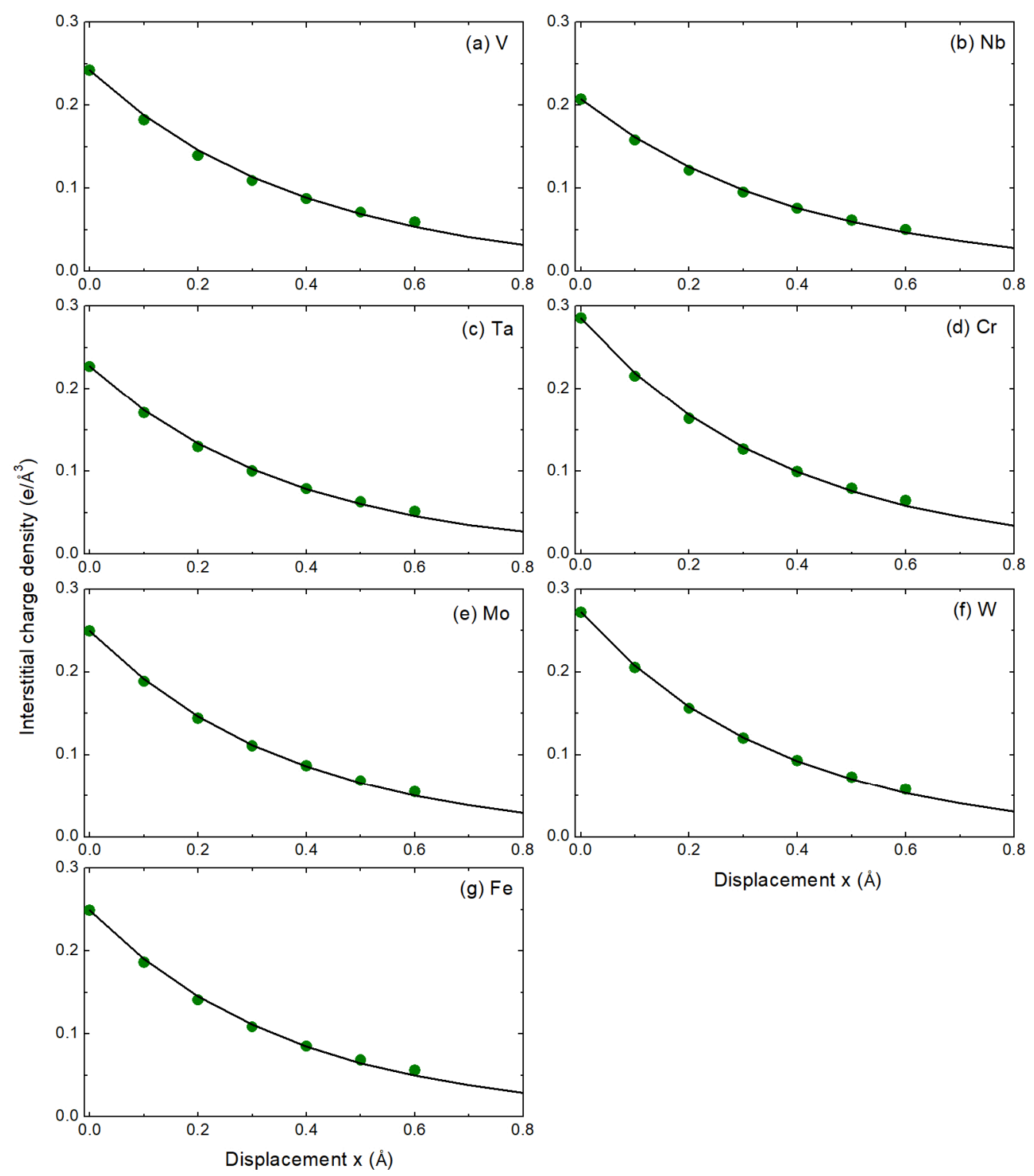

Displacement $x(\AA)$

Fig. 10. Valence charge density at tetrahedral sites in bcc transition metals from self-consistent DFT calculations, plotted as a function of the local displacement of metal atoms. The solid lines represent the best fitting of DFT data using Eq. (7). 


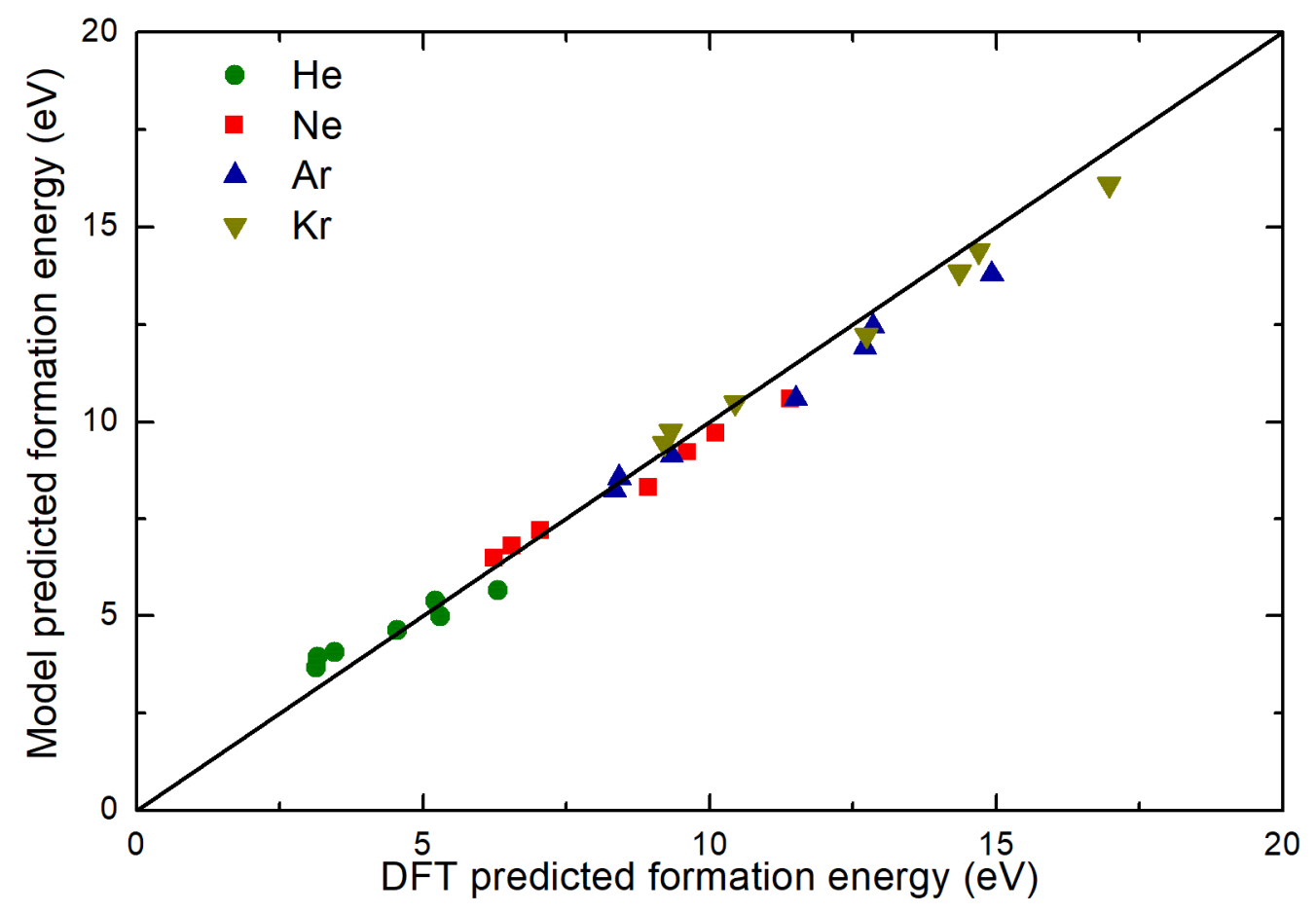

Fig. 11. Comparisons between model predicted and DFT calculated formation energies of noble gas atoms at tetrahedral sites in bcc transition metals. The solid line represents perfect agreement between DFT calculations and theory. 